Proceedings of the 43rd "Jaszowiec", International School and Conference on the Physics of Semiconductors, Wisła 2014

\title{
Sb Layers on $p$-GaN: UPS, XPS and LEED Study
}

\author{
M. Grodzicki, P. Mazur, J. Pers*, S. Zuber and A. Ciszewski \\ Institute of Experimental Physics, University of Wrocław, pl. M. Borna 9, 50-204 Wrocław, Poland
}

\begin{abstract}
The electronic structure of $p$-type $\mathrm{GaN}(0001)$ surfaces and its modification by antimony adsorption, and properties of $\mathrm{Sb} / \mathrm{GaN}(0001)$ interface, are presented in this report. The studies were carried out in situ by ultraviolet photoelectron spectroscopy, X-ray photoelectron spectroscopy, and low-energy electron diffraction. Thin Sb layers were deposited under ultrahigh vacuum conditions onto the substrate at room temperature. Electron affinity of clean $p$-GaN surface amounted to $3.0 \mathrm{eV}$. A small amount of $\mathrm{Sb}$ on $\mathrm{GaN}(0001)$ surface reduced the electron affinity to $1.9 \mathrm{eV}$. The work function of the Sb layer was equal to $4.4 \mathrm{eV}$. For the Schottky barrier height of the $\mathrm{Sb} / \mathrm{GaN}$ interface, the value of $2.50 \mathrm{eV}$ was obtained.
\end{abstract}

DOI: $10.12693 /$ APhysPolA.126.1128

PACS: 81.05.Dz, 68.37.Ps, 68.37.Ef, 68.37.-d, 81.10.Bk, 81.07.-b

\section{Introduction}

Gallium nitride (GaN), a wide band-gap semiconductor, is very attractive to electronic technology for devices and components like visible and UV lasers, light-emitting diodes, high temperature and frequency detectors, and transistors [1]. Antimony is an important element for electronics and optoelectronics technologies owing to its good thermal conductivity and a high oxidation- and corrosion resistance. It is also used as surfactant for better control of elementary growth processes responsible for the III-V semiconductor fabrication [2].

In our investigation antimony was used as a material for the interface formation on the GaN substrate and for changing the electronic properties of the semiconductor. Results of the $\mathrm{Sb} / \mathrm{GaN}$ interface formation after $\mathrm{Sb}$ deposition from vapor and morphological changes at the GaN surface caused by post-deposition annealing are described. We found that a small amount of $\mathrm{Sb}$, which remained on GaN surface after the annealing process, led to a substantial lowering of the electron affinity of the semiconductor's surface. The observed effect can be very helpful in producing the ohmic contact or heterojunction on the $p$-GaN substrate.

\section{Experimental details}

Samples, around $8 \times 4 \mathrm{~mm}^{2}$ in size, were cut out from a $\mathrm{GaN}(0001) / \mathrm{Al}_{2} \mathrm{O}_{3}$ wafer (TDI Inc.) and mounted on molybdenum plates. They consisted of atomically flat, (0001)-oriented, $p$-type, Mg-doped $\left(N_{a}=10^{18} \mathrm{~cm}^{-3}\right)$, and $10 \mu \mathrm{m}$ thick epitaxial GaN layers grown on a polished $\mathrm{Al}_{2} \mathrm{O}_{3}(0001)$ template. The substrates, $\mathrm{Sb} / \mathrm{GaN}$ interfaces and the $\mathrm{Sb}$ films were characterized by X-ray photoelectron spectroscopy (XPS) using an $\mathrm{Mg} K_{\alpha}(1253.6 \mathrm{eV})$ radiation source, and by ultraviolet photoelectron spectroscopy (UPS) using the He(I) line (21.2 eV) from a DC

*corresponding author; e-mail: jpers@ifd.uni.wroc.pl discharged lamp; additional analyses were carried out by low energy electron diffraction (LEED). These techniques were housed in an ultrahigh vacuum (UHV) chamber with the base pressure $\leq 1 \times 10^{-10}$ Torr. Photoelectrons were collected with a hemispherical electron energy analyzer (Phoibos 100, Specs) with $0.1 \mathrm{eV}$ steps. Optical axis of the analyzer entrance was normal to the substrate surface. The position of the Fermi level $\left(E_{\mathrm{F}}\right)$ was measured on a clean Au sample. The XPS and UPS spectra were analyzed using the Casa XPS software. The presence of carbon and oxygen was monitored using the AES $\mathrm{C}(K L L) \mathrm{O}(K L L)$ lines. Before measurements, the GaN samples were ex situ degreased in alcohol, then washed in distilled water and dried in air, next the substrate surface was cleaned in situ by cycles of annealing at $800{ }^{\circ} \mathrm{C}$ in order to remove surface oxides and carbon contamination. This was done by electron bombardment of the substrate from the backside. The Sb (99.999\% purity) was evaporated from an electron beam evaporator under an operating pressure lower than $1 \times 10^{-9}$ Torr. Ion current of the $\mathrm{Sb}$ molecular beam was used to control the efficiency of evaporation. Average coverage of Sb layers was quantified by the XPS method [3], the one-monolayer thickness was taken as equal to the diameter of an Sb atom. During experiments the residual gas pressure in the chamber was controlled by a quadrupole mass spectrometer. All the measurements were done at room temperature (RT).

\section{Results and discussion}

After in situ annealing the substrate several times at $800^{\circ} \mathrm{C}$, the Ga-rich surface of $\mathrm{GaN}(0001)$ with a very small amount of residual oxygen was obtained. The AES $\mathrm{C}(K K L)$ line was no longer present in the wide spectrum. The Ga $3 d$ line at $19.5 \mathrm{eV}$ had the full width at half-maximum (FWHM) equal to $2.1 \mathrm{eV}$. Its deconvolution into three components gave attributes of $\mathrm{Ga}^{-}$ $\mathrm{N}$ and $\mathrm{Ga}-\mathrm{O}$ bonds, and metallic Ga. The UPS spectrum of valence band is typical of a semiconductor (see Fig. 1, curve 1). Position of the binding energy at valence band maximum (VBM) is situated at $1.5 \mathrm{eV}$ 
below the $E_{\mathrm{F}}$, as determined from UPS measurements by extrapolation of the line fit to the leading edge of the spectrum. The VBM lies at $18 \mathrm{eV}$ above the $\mathrm{Ga} 3 d$ core level, which is in good agreement with the value reported by Bermudez [4]. The electron affinity of the GaN(0001) surface amounts to $3.0 \mathrm{eV}$, as calculated from the relationship $\chi=h v-W-E_{\mathrm{g}}$, where $h v=21.2 \mathrm{eV}$ is the energy of photons, the $W=14.8 \mathrm{eV}$ is the width of recorded spectrum measured as the energy difference between the VBM and the cut-off threshold of the spectrum, and the $E_{\mathrm{g}}=3.4 \mathrm{eV}$ is the band gap of the semiconductor.

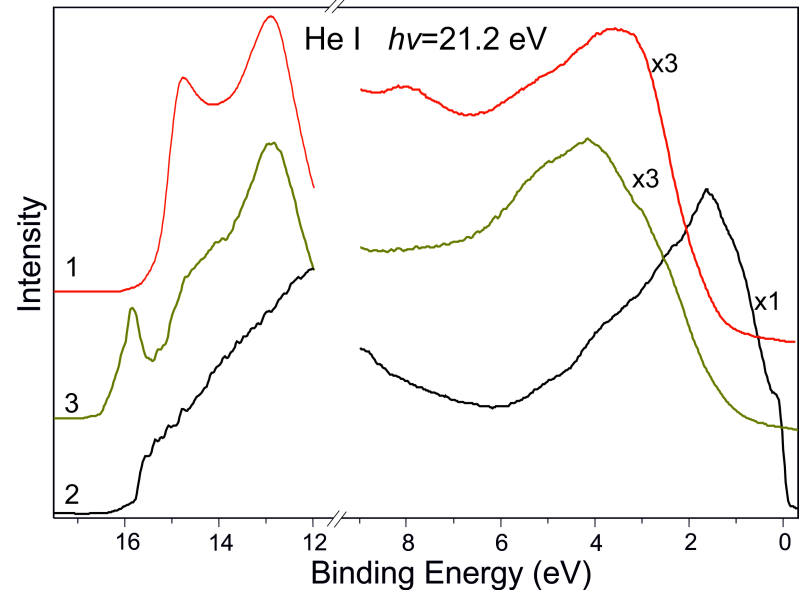

Fig. 1. UPS valence band spectra for (1) the clean $p$-GaN, (2) $5 \mathrm{~nm}$ thick $\mathrm{Sb}$ layer deposited on the GaN(0001), (3) Antimonized $p$-GaN(0001) surface with about 0.2 monolayer $\mathrm{Sb}$ obtained after annealing (2) at $400{ }^{\circ} \mathrm{C}$.

LEED patterns, with sharp diffraction spots and a dark background, revealed a highly ordered surface of the $(1 \times 1)$ structure. Instead of normal order spots, a sextet of diffraction spots appeared in the hexagonal configuration, such as in our previous work [5]. XPS measurement showed that, after deposition of an Sb layer, the $\mathrm{Ga} 3 d$ line shifted toward the high binding energy to reach $20.5 \mathrm{eV}$ and diminished with increase of evaporation time, while the signal from the $\mathrm{Sb} 4 d$ peak at $30 \mathrm{eV}$ increased. The Schottky barrier height (SBH) at the $\mathrm{Sb} / \mathrm{GaN}$ interface was determined from XPS $[6,7]$. The SBH for the $p$-type semiconductor can be calculated from the formula $\varphi_{\mathrm{B}}=E_{\mathrm{Ga} 3 d}^{m}-\Delta E$, where the $E_{\mathrm{Ga} 3 d}^{m}$ is the position of the $\mathrm{Ga} 3 d$ core level line as it is shifted after $\mathrm{Sb}$ deposition. The $\Delta E=18 \mathrm{eV}$ defines the initial binding energy of the $\mathrm{Ga} 3 d$ core level with respect to the VBM as mentioned above. SBH of the $\mathrm{Sb} / \mathrm{GaN}$ interface, as calculated basing on these data, amounts to $2.50 \mathrm{eV}$.

For the relatively thick $\mathrm{Sb}$ layers (of about $5 \mathrm{~nm}$ ), where spectral lines of substrate have almost disappeared, the band reveals a metallic character (see Fig. 1, curve 2). A high density of states occurs in the range between the $E_{\mathrm{F}}$ and the VBM. The work function of the $\mathrm{Sb}$ layers was calculated from the expression $\varphi_{\mathrm{m}}=h v-W$, where $W$ was estimated to be $16.8 \mathrm{eV}$. The resulting value was $4.4 \mathrm{eV}$. This value is consistent with literature data, where it ranges between 4.0 and $4.56 \mathrm{eV}[8,9]$. No diffraction pattern was observed for Sb layers. This indicates that the antimony layers had a grainy structure beginning from the earliest stages of growth.

The effect of the presence of Sb atoms on the electronic properties of the substrate was investigated. To achieve the objective the interface was short annealed at $400^{\circ} \mathrm{C}$ which led to incorporation of a small amount of Sb into the GaN surface. The UPS spectra indicate recovery of the valence band typical of a semiconductor from that of metals (curve 3 in Fig. 1) The position of VBM lies at $1.6 \mathrm{eV}$ below the $E_{\mathrm{F}}$, and the width $W$ of the recorded spectrum is $15.9 \mathrm{eV}$. Based on these values the electron affinity of the antimonized $\mathrm{GaN}(0001)$ surface was obtained to be $1.9 \mathrm{eV}$. The XPS spectrum of Ga $3 d$ (see Fig. 2) is shifted by $0.6 \mathrm{eV}$ from the position $20.5 \mathrm{eV}$ toward the low binding energy with its FWHM at $2.1 \mathrm{eV}$; the position of $\mathrm{Sb} 4 d$ remains unchanged but the FWHM is slightly increased from 2.3 to $2.4 \mathrm{eV}$. LEED patterns were the same as for the clean substrate, except that the diffraction spots for the low electron energy were slightly less sharp.

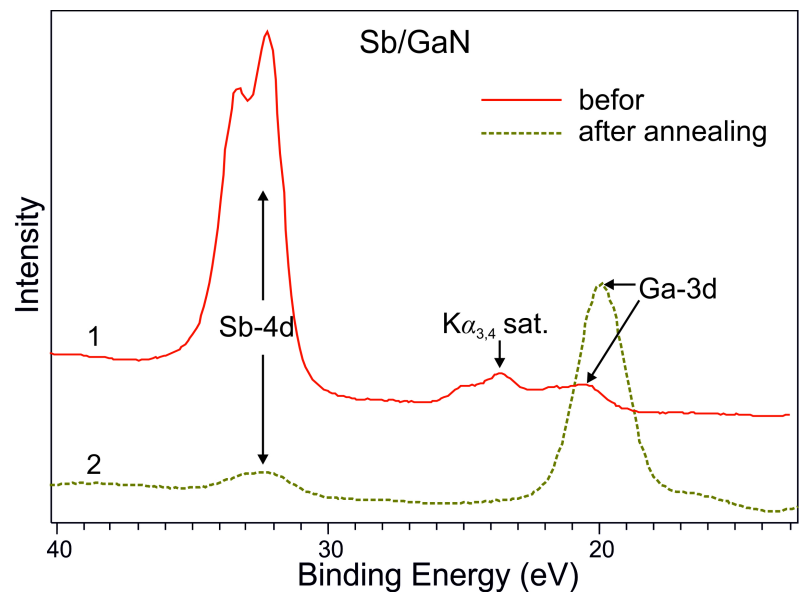

Fig. 2. The $\mathrm{Sb} 4 d$ and $\mathrm{Ga} 3 d$ XPS spectra from $\mathrm{Sb} / \mathrm{GaN}(0001)$ interfaces where: (1) thickness of $\mathrm{Sb}$ layer was $5 \mathrm{~nm}$, (2) thickness of Sb layer was 0.2 monolayer obtained after annealing $(1)$ at $400{ }^{\circ} \mathrm{C}$.

We elaborated the energy diagram of the $p$-GaN shown in Fig. 3. For this purpose we determined the position of the $E_{\mathrm{F}}$ relative to the VBM in bulk GaN. We have done the calculation for RT by data available in [10]. The result of $0.1 \mathrm{eV}$ indicates that the Fermi level is only $0.1 \mathrm{eV}$ above the VBM in the bulk. We conclude that the downward band bending is $1.4 \mathrm{eV}$ at the clean surface of the semiconductor. Let us note the slight shift of the VBM between the clean $p$-GaN(0001) surface and the surface of the substrate with small amounts of Sb. The difference in VBM value amounts to $0.1 \mathrm{eV}$. The vacuum level is lowered by $1.2 \mathrm{eV}$. The presence of antimony on the surface leads to the lowering of the electron affinity from 3.0 to $1.9 \mathrm{eV}$. 


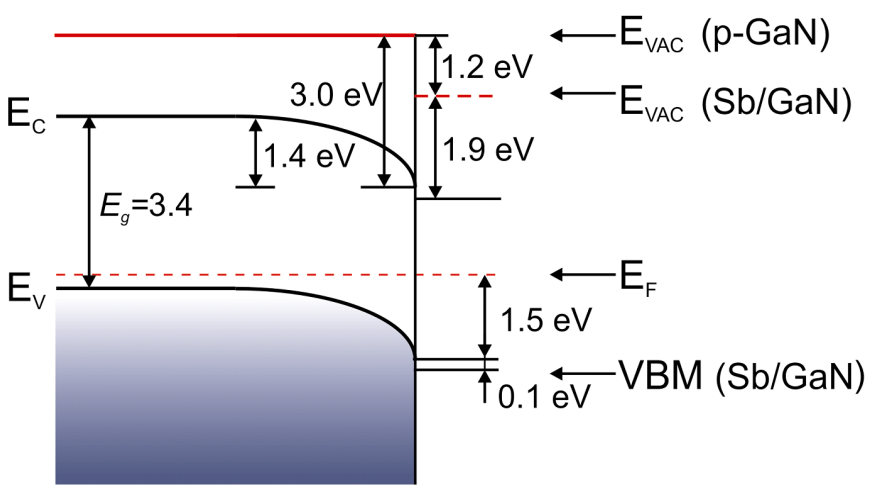

Fig. 3. Schematic energy diagram of the $p-\mathrm{GaN}(0001)$ surface showing the Sb-induced lowering effect of electron affinity.

\section{Conclusions}

Combined surface-analysis techniques of UPS, XPS and LEED were used to investigate chemical and electrical properties of the $\mathrm{Sb} / \mathrm{GaN}(0001)$ interface. The electron affinity is $3.0 \mathrm{eV}$ and the downward band bending is $1.4 \mathrm{eV}$ at the clean surface of $p-\mathrm{GaN}(0001)$. Diffraction pattern exhibits a highly ordered surface of the $(1 \times 1)$ structure. Incorporation of a small amount of $\mathrm{Sb}$ into the $p$-GaN(0001) surface leads to a substantial decrease of the electron affinity to $1.9 \mathrm{eV}$. After the procedure LEED patterns remain the same like those for the clean surface of the semiconductor. The work function of the Sb layers equal to $4.4 \mathrm{eV}$ has been calculated from the obtained results. The XPS measurements have excluded a chemical interaction at the $\mathrm{Sb} / \mathrm{GaN}$ interface for the RT deposited Sb layers. The Schottky barrier height of the $\mathrm{Sb} / \mathrm{GaN}$ interface, as calculated from the data acquired by using XPS and UPS, is equal to $250 \mathrm{eV}$.

\section{Acknowledgments}

The authors would like to thank Dr. Stanisław Surma for helpful discussions and his assistance during preparation of manuscript. The work was supported by the Wrocław Research Centre EIT + within the project "The Application of Nanotechnology in Advanced Materials" NanoMat (POIG.01.01.02-02-002/08) co-financed by the European Regional Development Fund (Operational Programme Innovative Economy, 1.1.2).

\section{References}

[1] Y.N. Saripalli, L. Pei, T. Biggerstaff, S. Ramachandran, G.J. Duscher, M. Johnson, Appl. Phys. Lett. 90, 204106 (2007)

[2] A.A. Gokhale, T.F. Kuech, M. Mavrikakis, J. Cryst. Growth 285, 146 (2005).

[3] S. Tanuma, C.J. Powell, D.R. Penn, Surf. Interface Anal. 11, 577 (1988).

[4] V.M. Bermudez, J. Appl. Phys. 80, 1190 (1996).

[5] M. Grodzicki, P. Mazur, S. Zuber, J. Brona, A. Ciszewski, Appl. Surf. Sci. 304, 20 (2014).

[6] J.R. Waldrop, R.W. Grant, Y.C. Wang, R.F. Davis, J. Appl. Phys. 72, 4757 (1992).

[7] Y.-J. Lin, C.-W. Hsu J. Electron. Mater. 33, 1037 (2004).

[8] E.A. Mechtly, in: Properties of Materials. Reference Data For Engineers Radio, Electronics, Computer, and Communications, 9th ed., Ed. W.M. Middleton, Butterworth-Heinemann, Woburn (MA) 2002, p. $4-1$.

[9] J. Speight, Lange's Handbook of Chemistry, 16th ed., McGraw-Hill Professional, Boston (MA) 2002, p. 4.

[10] V. Siklitsky, electronic archive: NSM Characteristics and Properties (http://www.ioffe.ru/SVA/NSM/). 